\title{
Interaction véhicule-voie-sol et vibrations dues aux trains
}

\section{Modélisations et vérifications expérimentales}

\author{
Lutz Auersch* — Marc Maldonado** \\ * BAM Institut Fédéral pour la Recherche et l'Essai des Matériaux \\ 87, Unter den Eichen - D-12200 Berlin, Allemagne \\ lutz.auersch-saworski@bam.de \\ ** GEM Laboratoire de Génie Civil et Mécanique \\ 1 Rue de la Noë, F-44321 Nantes cedex 3, France \\ marc.maldonado@univ-angers.fr
}

RÉSUMÉ. Cet article présente plusieurs modèles numériques pour l'étude des phénomènes vibratoires lors du passage d'un train. Les modèles permettent d'estimer la propagation des ondes et les réceptances pour le sol et la voie. Le sol multicouche et le couplage voie-sol sont traités par une (double) intégration sur les nombres d'onde. Les raideurs dynamiques de la voie et $d u$ véhicule sont combinées et les forces d'excitation roue-rail dues aux irrégularités de la voie et des roues sont calculées. Ces forces d'excitation permettent de simuler les vibrations du sol lors du passage d'un train. Les modèles sont validés par comparaison avec des mesures sur deux sites, en France et en Allemagne. Les vibrations calculées correspondent bien aux vibrations mesurées.

ABSTRACT. This contribution presents models that are necessary to calculate the vibrations due to the passage of a train. The models allow to calculate the propagation of the waves and the receptances of the soil and the track. The layered soil and the coupling with the track are treated by a (double) integration in wavenumber domain. The dynamic stiffnesses of the track and vehicle are combined and the excitation forces due to the irregularities of the track and the wheel are calculated. Finally, these excitation forces are used to simulate the ground vibration of a passing train. All these models are validated by a number of different measurements at two sites in France and Germany.

MOTS-CLÉS : ondes du sol multicouche, irrégularités et forces roue-rail.

KEYWORDS: wave propagation, layered soil, wheel-rail irregularities and forces.

DOI:10.3166/EJCM.20.257-280 @ 2011 Lavoisier, Paris 


\section{Introduction}

Les phénomènes vibratoires rencontrés lors du passage d'un train roulant à « faible vitesse » sur une voie ferrée sont généralement liés aux irrégularités du contact rouerail. Ces dernières génèrent des forces d'interaction qui induisent des vibrations sur la voie, se transmettant finalement dans le sol. La modélisation de ces phénomènes nécessite d'une part de prendre en compte la propagation des ondes dans le sol, et d'autre part de s'intéresser à la source de l'excitation, notamment en caractérisant l'interaction véhicule-voie.

Pour modéliser la propagation des ondes dans le sol, une approche semi-analytique bien connue de la sismologie est retenue dans cet article (Kausel et al., 1981; Wolf, 1985). Pour chaque fréquence, les équations sont écrites dans le domaine des nombres d'onde (ou domaine fréquences-nombres d'onde) et une matrice de raideur globale caractérisant le sol est obtenue par assemblage des matrices de raideur pour chaque couche du sol. L'intégration sur les nombres d'onde donne alors les vibrations du sol pour le cas d'une source ponctuelle ou circulaire fixe. La propagation des ondes caractéristiques pour les sols stratifiés a été analysée par cette méthode (Girardi, 1981; Jones, 1987; Auersch, 1994; Laghrouche, 1996; Sheng, 2001).

Ces dix dernières années, cette méthode d'intégration a été mise en œuvre dans de nombreuses contributions pour calculer les vibrations de la voie et du sol dues à des sources mobiles (Barros et al., 1994; Aubry et al., 1994; Dietermann et al., 1996; Grundmann et al., 1999; Lefeuve-Mesgouez, 1999; Sheng, 2001; Takemiya, 2003; Auersch, 2008b). Il existe d'autres méthodes pour simuler des sources mobiles, notamment des méthodes simplifiées (Huber, 1988; Krylov, 2001), la « thin layer method » (Jones et al., 1996), l'utilisation de la méthode des éléments finis (Madshus et al., 2000; O’Brien et al., 2005) ou des éléments de frontière (Galvin et al., 2009) dans le domaine de temps ou fréquences (voir un résumé dans Hung et al. (2001)). La majorité de ces contributions s'intéresse à l'effet des trains à grande vitesse roulant sur des sols "souples", i.e. pour lesquels la vitesse de propagation des ondes dans le sol peut être plus faible que celle du train (régime super Rayleigh). Dans ce cas, généralement seules les sources quasi statiques sont considérées.

Pour les deux configurations réelles qui sont analysées dans cet article, la vitesse du train n'a pas d'effet notable et on peut ainsi utiliser une formulation dans le domaine des nombres d'onde pour des sources dynamiques qui seront alors supposées fixes. Notons qu'il existe d'autres méthodes «simples » pour la prédiction des vibrations du sol dues aux trains en considérant des sources immobiles, par exemple des méthodes empiriques (Fujikake, 1986) ou approchées (Yokoyama et al., 2005), développées par l'Institut de Recherche Ferroviaire du Japon et qui semblent présenter des résultats réalistes pour les sols stratifiés. De même, Nelson et al. (1987) ont développé une méthode semi-expérimentale avec une fonction de transfert du sol spécifique pour le trafic ferroviaire qui est maintenant une méthode de prédiction officielle aux ÉtatsUnis (Hanson et al., 2006). 
Concernant l'interaction véhicule-voie, elle a d'abord été étudiée à l'aide de modèles simples (Fryba, 1972; Knothe et al., 1993). Dans ces études la modélisation de l'ensemble véhicule-voie est très détaillée. En revanche, la contribution du sol (couplage) n'est parfois pas prise en compte ou modélisée de façon très simplifiée (fondation de Winkler), et la propagation dans le sol n'est pas abordée. D'un autre côté, lorsque les modèles sont plus élaborés pour le sol, l'interaction véhicule-voie et l'excitation dynamique de la voie sont modélisées à l'aide de paramètres simples et peu réalistes. Enfin, certains travaux proposent des modélisations pour les deux aspects "excitation" et "propagation" mais avec une résolution découplée (Saedeleer et al., 1998). Finalement, rares sont les auteurs qui s'attachent à prendre en compte avec précision l'ensemble des phénomènes véhiculevoie-sol, par exemple (Girardi, 1981; Auersch, 1990; Jones et al., 1996; van den Broek, 2001). Il existe maintenant quelques campagnes expérimentales pour vérifier les modèles véhicule-voie-sol (Auersch, 1988; Nielsen, 1993; Fröhling, 1997; Adolfsson et al., 1999; Picoux, 2002; Auersch, 2005b; Lombaert et al., 2006; Galvin et al., 2009), chacune se focalisant généralement sur un aspect du problème (excitation ou propagation).

Finalement, une étude complète de l'interaction véhicule-voie-sol fait appel à différentes compétences, notamment la géotechnique pour le sol, le génie civil pour la voie et la construction de machines pour le véhicule. Elle nécessite donc une comparaison avec des mesures faites à la fois sur le sol, la voie et le véhicule. Cet article présente une confrontation modèles-mesures sur ces trois points, se basant sur des études de l'Ecole Centrale de Nantes (Maldonado, 2008) et de l'Institut Fédéral BAM (Auersch et al., 2001). L'originalité de ces travaux est donc principalement située dans l'approche globale de l'interaction véhicule-voie-sol et sa validation expérimentale.

L'article se décompose en cinq sections. La section 2 présente les modèles pour le sol, pour le système voie-sol et pour l'interaction véhicule-voie-sol. La section 3 décrit les deux campagnes expérimentales qui ont eu lieu en France et en Allemagne. La section 4 présente les résultats pour les vibrations du sol à l'aide des fonctions de transfert sur deux sites (à Nantes et à Würzburg). L'influence de la voie, dans le cas d'une pose sur dalle (Nantes) est analysée numériquement dans la section 5. La section 6 donne les principaux résultats pour l'interaction véhicule-voie pour le tramway à Nantes et le train à Würzburg. Enfin la section 7 présente une comparaison modèles-mesures pour les vibrations du sol lors du passage d'un train.

\section{Modèles pour le sol, la voie et le véhicule}

Les principales notations adoptées dans l'analyse de l'interaction véhicule-voiesol sont récapitulées dans le tableau 1. 


\begin{tabular}{|c|c|c|}
\hline$a$ & $\mathrm{~m}$ & demi-largeur de la voie \\
\hline$d$ & $\mathrm{~m}$ & épaisseur d'une couche du sol \\
\hline$d_{D}$ & $\mathrm{~m}$ & épaisseur de la dalle \\
\hline$D$ & & coefficient d'amortissement (hystérétique) du sol \\
\hline$E$ & $\mathrm{Nm}^{-2}$ & module de Young \\
\hline$E I$ & $\mathrm{Nm}^{2}$ & raideur en flexion (rails et dalle de béton) \\
\hline$f$ & $\mathrm{~Hz}$ & fréquence \\
\hline $\mathbb{F}, F_{z z}$ & $\mathrm{~m}^{3} N^{-1}$ & flexibilité (réceptance) du sol (domaine transformé) \\
\hline$F$ & $\mathrm{~N}$ & force \\
\hline$F_{T V}$ & $\mathrm{~N}$ & force roue-voie \\
\hline$F^{\prime}$ & $\mathrm{Nm}^{-1}$ & force par unité de longueur de voie \\
\hline$G_{0}$ & $\mathrm{Nm}^{-2}$ & module de cisaillement, partie élastique \\
\hline$G$ & $\mathrm{Nm}^{-2}$ & complexe module de cisaillement $G=G_{0}(1+i 2 D)$ \\
\hline$H$ & $\mathrm{~m}^{3} N^{-1}$ & fonction de transfert du sol \\
\hline$H_{V S}$ & $\mathrm{~m}^{2} N^{-1}$ & fonction de transfert voie-sol (déplacements) \\
\hline$k_{x}, k_{y}$ & $\mathrm{~m}^{-1}$ & nombres d'ondes \\
\hline & $\mathrm{Nm}^{-3}$ & matrice de raideur du sol \\
\hline $\mathbb{K}_{\mathbb{C}}, K_{i j}$ & $\mathrm{Nm}^{-3}$ & matrice de raideur d'une couche du sol \\
\hline$K_{S}$ & $\mathrm{Nm}^{-2}$ & raideur du sol (interface voie-sol) \\
\hline$K_{V}$ & $\mathrm{Nm}^{-2}$ & raideur de la voie \\
\hline$K_{V S}$ & $\mathrm{Nm}^{-1}$ & raideur de la voie sur le sol \\
\hline$K_{T}$ & $\mathrm{Nm}^{-1}$ & raideur du véhicule \\
\hline$m^{\prime}$ & $\mathrm{kgm}^{-1}$ & masse de la dalle par unité de longueur de la voie \\
\hline$m_{R}$ & $\mathrm{~kg}$ & masse d'un essieu \\
\hline$P$ & $\mathrm{~N}$ & force d'excitation \\
\hline$r$ & $\mathrm{~m}$ & distance du point d'excitation \\
\hline$s$ & $\mathrm{~m}$ & irrégularité de la voie et des roues \\
\hline$\vec{\sigma}_{C}$ & $\mathrm{Nm}^{-2}$ & vecteur contrainte sur une couche du sol \\
\hline$\sigma_{z z}$ & $\mathrm{Nm}^{-2}$ & contrainte verticale sur la surface du sol \\
\hline$T_{V S}$ & & fonction de transfert voie-sol (forces) \\
\hline$u$ & $\mathrm{~m}$ & déplacement \\
\hline$\vec{u}$ & $\mathrm{~m}$ & vecteur déplacement du sol \\
\hline$\vec{u}_{C}$ & $\mathrm{~m}$ & vecteur déplacement d'une couche du sol \\
\hline$u_{T}$ & $\mathrm{~m}$ & déplacement vertical de la roue \\
\hline$u_{V}$ & $\mathrm{~m}$ & déplacement vertical de la voie \\
\hline$y, x$ & & axe longitudinal et transversal de la voie ferrée \\
\hline$v_{S}$ & $\mathrm{~ms}^{-1}$ & vitesse de l'onde de cisaillement \\
\hline$v_{P}$ & $\mathrm{~ms}^{-1}$ & vitesse de l'onde de compression \\
\hline$v$ & $\mathrm{~ms}^{-1}$ & vitesse verticale du sol \\
\hline$\rho$ & $\mathrm{kgm}^{-3}$ & masse volumique \\
\hline$\omega$ & $\operatorname{rads}^{-2}$ & pulsation \\
\hline
\end{tabular}

Tableau 1. Principales notations 


\subsection{Vibrations du sol}

\subsubsection{Modélisation d'un sol multicouche dans le domaine des nombres d'onde}

Dans ce modèle, on suppose que le sol est constitué de plusieurs couches horizontales et homogènes. Chaque couche satisfait l'équation élastodynamique de Navier :

$$
v_{P}^{2} \vec{\nabla}(\vec{\nabla} \cdot \vec{u})+v_{S}^{2} \vec{\nabla} \wedge(\vec{\nabla} \wedge \vec{u})=\ddot{\vec{u}}
$$

avec la vitesse de l'onde de compression $v_{P}$ et la vitesse de l'onde de cisaillement $v_{S}$. La solution est déterminée dans le domaine des fréquences $f$ et des nombres d'onde $k$ à l'aide d'une double transformée de Fourier suivant les deux directions horizontales $(x$ et $y)$.

Une matrice de raideur $\mathbb{K}_{\mathbb{C}}$ symétrique est établie pour chaque couche selon les formules suivantes :

$$
\begin{aligned}
\frac{K_{11}}{A} & =\frac{K_{33}}{A}=\frac{1}{t} C_{s} S_{t}+s S_{s} C_{t} \\
\frac{K_{22}}{A} & =\frac{K_{44}}{A}=t C_{s} S_{t}+\frac{1}{s} S_{s} C_{t} \\
\frac{K_{12}}{A} & =-\frac{K_{34}}{A}=\frac{3-t^{2}}{1+t^{2}}\left(1-C_{s} C_{t}\right)+\frac{1+2 s^{2} t^{2}-t^{2}}{s t\left(1+t^{2}\right)} S_{s} S_{t} \\
\frac{K_{13}}{A} & =-s S_{s}-\frac{1}{t} S_{t} \\
\frac{K_{14}}{A} & =-\frac{K_{23}}{A}=C_{s}-C_{t} \\
\frac{K_{24}}{A} & =-\frac{1}{s} S_{s}-t S_{t}
\end{aligned}
$$

où les abréviations suivantes sont utilisées :

$$
\begin{array}{ll}
s=-i \sqrt{1-\left(\frac{\omega}{k v_{P}}\right)^{2}} & t=-i \sqrt{1-\left(\frac{\omega}{k v_{S}}\right)^{2}} \\
C_{s}=\cos k s d & C_{t}=\cos k t d \\
S_{s}=\sin k s d & S_{t}=\sin k t d \\
N=2\left(1-C_{s} C_{t}\right)+\left(s t+\frac{1}{s t}\right) S_{s} S_{t} & A=\frac{\left(1+t^{2}\right) k G}{N}
\end{array}
$$

Pour plus de détails, voir Kausel et al. (1981) ou Wolf (1985). La matrice de raideur $\mathbb{K}_{\mathbb{C}}$ d'une couche permet de relier les contraintes $\vec{\sigma}_{C}$ aux déplacements $\vec{u}_{C}$ sur les deux surfaces horizontales limitant la couche considérée :

$$
\vec{\sigma}_{C}\left(k_{x}, k_{y}, \omega\right)=\mathbb{K}_{\mathbb{C}}\left(k_{x}, k_{y}, \omega\right) \vec{u}_{C}\left(k_{x}, k_{y}, \omega\right)
$$


L'assemblage des matrices de raideurs pour chaque couche et pour le massif semiinfini fournit une matrice de raideur globale $\mathbb{K}$, puis par inversion on obtient la matrice de flexibilité $\mathbb{F}$ pour le sol multicouche :

$$
\mathbb{K}^{-1}=\mathbb{F}
$$

La composante $F_{z z}$ correspond à la fonction de transfert du sol (en surface) dans le domaine fréquence-nombres d'onde, soit :

$$
H\left(k_{x}, k_{y}, \omega\right)=F_{z z}
$$

avec la relation liant le déplacement vertical à la contrainte verticale dans le domaine transformé :

$$
u\left(k_{x}, k_{y}, \omega\right)=H\left(k_{x}, k_{y}, \omega\right) \sigma_{z z}\left(k_{x}, k_{y}, \omega\right)
$$

\subsubsection{Intégration dans le domaine des nombres d'onde}

La réponse vibratoire verticale du sol à une excitation harmonique verticale s'appliquant en surface est calculée par l'intégration sur les nombres d'onde :

$$
u(x, y, \omega)=\frac{1}{(2 \pi)^{2}} \int_{-\infty}^{+\infty} \int_{-\infty}^{+\infty} H\left(k_{x}, k_{y}, \omega\right) \sigma_{z z}\left(k_{x}, k_{y}, \omega\right) e^{i\left(k_{x} x+k_{y} y\right)} \mathrm{d} k_{x} \mathrm{~d} k_{y}
$$

Dans le cas d'une force ponctuelle $P$ on a :

$$
\sigma_{z z}\left(k_{x}, k_{y}\right)=P
$$

et la réponse du sol devient :

$$
u(x, y, \omega)=\frac{P}{(2 \pi)^{2}} \int_{-\infty}^{+\infty} \int_{-\infty}^{+\infty} H\left(k_{x}, k_{y}, \omega\right) e^{i\left(k_{x} x+k_{y} y\right)} \mathrm{d} k_{x} \mathrm{~d} k_{y}
$$

On peut aussi écrire en coordonnées polaires pour ce problème axisymétrique :

$$
u(r, \omega)=\frac{P}{2 \pi} \int_{0}^{+\infty} H(k, \omega) J_{0}(k r) \mathrm{d} k
$$

avec la fonction de Bessel $J_{0}$. Cette intégrale est évaluée numériquement avec la formule des rectangles. L'intervalle $\left(0, k_{\max }\right)$ et l'incrément $d k$ sont choisis en fonction des vitesses de l'onde de cisaillement dans les différentes couches et de l'ondulation de la fonction de Bessel. Comme autre possibilité, la transformée de Fourier inverse à deux dimensions peut être utilisée pour résoudre l'équation [10]. 


\subsection{Interaction voie-sol}

\subsubsection{Réceptance du sol}

La voie de largeur $2 a$, orientée suivant la direction $y$, est constituée de deux rails et d'une dalle de béton de raideur en flexion $E I$, de masse $m^{\prime}$. L'effort $F^{\prime}$ s'exerce sur les deux rails. L'équation du mouvement est donnée par :

$$
E I \frac{\partial^{4} u}{\partial y^{4}}+m^{\prime} \ddot{u}=F^{\prime}
$$

La raideur dynamique dans le domaine des fréquences et des nombres d'onde est alors :

$$
K_{V}\left(k_{y}, \omega\right)=E I k_{y}^{4}-m^{\prime} \omega^{2}
$$

La raideur dynamique du sol est calculée par l'intégrale (Auersch, 2008a) :

$$
\frac{1}{K_{S}\left(k_{y}, \omega\right)}=\frac{1}{2 \pi} \int_{-\infty}^{+\infty} H\left(k_{x}, k_{y}, \omega\right) p_{1}^{2}\left(k_{x}\right) \mathrm{d} k_{x}
$$

On suppose une distribution uniforme pour la force s'appliquant à l'interface voie-sol selon la direction transversale $x$, avec la transformée :

$$
p_{1}\left(k_{x}\right)=\frac{\sin k_{x} a}{k_{x} a}
$$

Les deux raideurs du sol et de la voie sont sommées pour former d'une part la fonction de transfert d'interaction pour les déplacements de la voie sur le sol :

$$
H_{V S}\left(k_{y}, \omega\right)=\frac{1}{K_{V}+K_{S}}
$$

et d'autre part la fonction de transfert d'interaction pour les forces voie-sol :

$$
T_{V S}\left(k_{y}, \omega\right)=\frac{K_{S}}{K_{V}+K_{S}}
$$

Enfin, les vibrations du sol sont calculées par une intégration numérique :

$u(x, y, \omega)=\frac{P}{(2 \pi)^{2}} \int_{-\infty}^{+\infty} \int_{-\infty}^{+\infty} H\left(k_{x}, k_{y}, \omega\right) T_{V S}\left(k_{y}, \omega\right) p_{1}\left(k_{x}\right) e^{i\left(k_{x} x+k_{y} y\right)} \mathrm{d} k_{x} \mathrm{~d} k_{y}$

Pour une source roulante de vitesse $v_{T}$, cette intégrale est modifiée comme suit :

$u(x, y *, \omega)=\frac{P}{(2 \pi)^{2}} \int_{-\infty}^{+\infty} \int_{-\infty}^{+\infty} H\left(k_{x}, k_{y}, \omega *\right) T_{V S}\left(k_{y}, \omega *\right) p_{1}\left(k_{x}\right) e^{i\left(k_{x} x+k_{y} y *\right)} \mathrm{d} k_{x} \mathrm{~d} k_{y}$ 
ou $y *$ et $\omega *$ sont donnés respectivement par $y-v_{T} t$ et $\omega-k_{y} v_{T}$. Des modifications similaires sont aussi possibles pour la receptance de la voie (équations [20] et [21]). Comme précisé dans l'introduction, les charges roulantes sont supposées fixes dans les calculs suivants. En effet, les différentes vitesses de propagation des ondes dans le rail et la voie (puis dans le sol) sont grandes vis-à-vis de la vitesse de déplacement des charges. Ainsi le déplacement du train n'est pas pris en compte.

\subsubsection{Réceptance de la voie}

Pour les déplacements de la voie on peut évaluer directement la fonction de transfert voie-sol :

$$
u(y, \omega)=\frac{P}{2 \pi} \int_{-\infty}^{+\infty} H_{V S}\left(k_{y}, \omega\right) e^{i k_{y} y} \mathrm{~d} k_{y}
$$

et pour le point d'application de l'excitation :

$$
u(0, \omega)=\frac{P}{2 \pi} \int_{-\infty}^{+\infty} H_{V S}\left(k_{y}, \omega\right) \mathrm{d} k_{y}
$$

C'est la réceptance de la voie, ou par inversion la raideur $K_{V S}$ de la voie sur le sol, qui est utilisée pour l'interaction véhicule-voie-sol.

Si l'on souhaite inclure des éléments additifs de la voie comme des traverses, des semelles entre les rails et les traverses, et d'autres matériaux isolants (par exemple, pose sur dalle flottante), on peut utiliser des modèles plus spécifiques : voir notamment le modèle multipoutre (Maldonado, 2008) pour la voie avec dalle béton, et la méthode des éléments finis et des éléments de frontière combinés (Auersch, 2005a) pour la modélisation de la voie ballastée.

\subsection{Interaction véhicule-voie}

L'interaction entre le véhicule et la voie est formulée dans le domaine fréquentiel à l'aide des raideurs dynamiques de la voie et du train. La raideur dynamique $K_{V}$ de la voie permet d'exprimer la force $F_{T V}$ exercée par le train sur la voie en fonction du déplacement de la voie $u_{V}$, voir figure 1 . On peut écrire :

$$
F_{T V}=K_{V S} u_{V}
$$

La raideur dynamique $K_{T}$ du train donne la force $-F_{T V}$, reliée au déplacement $u_{T}$ de la roue :

$$
-F_{T V}=K_{T} u_{T}
$$

Le système train-voie est excité par les irrégularités $s$ de la voie et des roues (rugosités). Les déplacements de la voie et d'une roue en contact sont liés à ces irrégularités selon la relation :

$$
u_{T}=u_{V}+s
$$




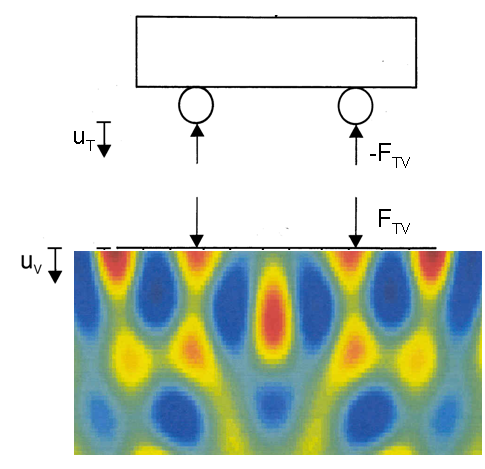

Figure 1. Problème de l'interaction véhicule-voie-sol

La combinaison des équations précédentes permet d'écrire les fonctions de transfert caractéristiques de l'interaction train-voie, à savoir pour les déplacements de la roue :

$$
\frac{u_{T}}{s}=\frac{K_{V S}}{K_{V S}+K_{T}}
$$

et pour les forces dues au train qui agissent sur la voie (et, en fin de compte, sur le sol) :

$$
\frac{F_{T V}}{s}=\frac{K_{T} K_{V S}}{K_{V S}+K_{T}}
$$

Il existe des méthodes et des modèles différents pour calculer la raideur dynamique du train, par exemple les modèles multimasses, ou des modèles qui incluent les rotations d'essieux. Le modèle le plus simple est celui considérant un essieu de masse $m_{R}$, dont la raideur dynamique est donnée par :

$$
K_{T}=-m_{R} \omega^{2}
$$

\subsection{Simulations des vibrations dues au passage d'un train}

La propagation des ondes dues au passage d'un train est calculée en superposant les contributions des forces dynamiques pour chaque essieu, supposées non corrélées :

$|u(x, \omega)|=\sqrt{\sum_{i=1}^{n}\left|\frac{u}{P}\left(x, y_{i}, \omega\right)\right|^{2}\left|F_{T V}(\omega)\right|^{2}}=\sqrt{\sum_{i=1}^{n}\left|\frac{u}{P}\left(x, y_{i}, \omega\right)\right|{ }^{2}}\left|F_{T V}(\omega)\right|$

Les positions des essieux sont représentées par les coordonnés $y_{i}$. La position du train pour laquelle les vibrations sont maximales est utilisée ici, donc le milieu du train est 
positionné sur l'ordonnée $y=0$. Étant donné l'homogénité et la symétrie du problème, la réponse d'un point de coordonnées $(x, y)=(x, 0)$, due à une source situé à $\left(0, y_{i}\right)$ peut être calculée comme la réponse à la position $\left(x, y_{i}\right)$ due à la source de coordonnées $(0,0)$. Les forces sont calculées avec les équations établies pour l'interaction véhicule-voie dans la section 2.3 (équation [26]). Les amplitudes du sol sont calculées à l'aide de la fonction de transfert voie-sol, voir l'équation [18] (section 2.2).

Dans Auersch (2010) une méthode simple a été développée à l'aide de la fonction de transfert du sol définie par l'équation [11] (section 2.1) et l'influence de la voie qui sera présentée dans la section 5. Cette approche donne des résultats similaires à ceux obtenus avec l'équation [28] et la fonction de transfert du sol théorique. Elle permet aussi d'utiliser les fonctions de transfert du sol mesurées et les résultats correspondants sont présentés dans la section 7 .

\section{Campagnes expérimentales}

Deux campagnes complémentaires sont utilisées pour valider les modèles. L'Ecole Centrale de Nantes et l'INRETS ont réalisé une campagne expérimentale à Nantes, où deux tramways différents ont été étudiés (Maldonado et al., 2009). Le premier type de tramway (plancher bas) est constitué de roues indépendantes, l'autre type de tramway possède des essieux. Les tramways roulent sur quatre voies différentes, une voie ballastée, une voie avec une dalle de béton (non isolante), une pose isolante (semelles isolantes sous les rails) et une voie avec une dalle isolante (dalle flottante). Dans la contribution présentée, seul le tramway avec des essieux est étudié pour une voie sur dalle béton.

La deuxième campagne expérimentale a été faite par l'Institut Fédéral (BAM). Elle concerne les trains de voyageurs près de Würzburg (Auersch et al., 2001). Trois configurations différentes sont évaluées simultanément : une voie ballastée sur le sol, une voie ballastée sur un pont et une voie sur dalle béton dans un tunnel. Dans la présente contribution, seule la voie ballastée sur sol est analysée.

Les deux campagnes comportent les essais suivants. D'abord, à l'aide d'une excitation du sol, vibratoire ou impulsive, la vitesse de l'onde de surface (et sa dispersion) et la fonction de transfert sont déterminées. Ensuite, l'excitation impulsive du rail permet d'estimer la fonction de transfert voie-sol et la réceptance de la voie. Par ailleurs, les rugosités de la voie sont mesurées. Simultanément, les vibrations du sol sont mesurées pour plusieurs distances de la voie. Lors du passage du train, les accélérations du véhicule sont mesurées. Lors des mesures, le tramway circule aux vitesses valant $20,30,40$ et $50 \mathrm{~km} / \mathrm{h}$, et le train voyageur à $40,63,80,100,125,140$ et $160 \mathrm{~km} / \mathrm{h}$.

\section{Résultats théoriques et expérimentaux : la fonction de transfert du sol}

Les modèles des sites sont établis itérativement par comparaison sur la vitesse de l'onde de surface pour différentes fréquences (ajustement à l'aide de la dispersion me- 
surée de la vitesse de l'onde superficielle de Rayleigh, utilisant la méthode de Lai et al. (2002), voir Maldonado (2008)). Les données sont ensuite affinées pour l'amortissement et la raideur des couches à l'aide des fonctions de transfert par « essai et erreur ». Pour le sol du site de Würzburg on considère un sol moyen raide $\left(v_{S}=250\right.$ $\mathrm{m} / \mathrm{s})$ sur une épaisseur de $10 \mathrm{~m}$, et reposant sur un sous-sol raide $\left(v_{S}=1000 \mathrm{~m} / \mathrm{s}\right)$. Pour les autres paramètres des valeurs usuelles sont choisies :

$$
\begin{array}{r}
\rho_{1}=\rho_{2}=2000 \mathrm{~kg} / \mathrm{m}^{3} \\
\nu_{1}=\nu_{2}=0.33
\end{array}
$$

La fonction de transfert du sol est calculée à l'aide de l'équation [11] et présentée sur la figure 2(a) pour les distances allant de 4 à $64 \mathrm{~m}$. La mobilité de transfert (rapport de la vitesse sur la force d'excitation), augmente avec la fréquence. Un maximum est atteint pour chaque courbe, puis pour les fréquences hautes la mobilité s'atténue. Ce résultat est caractéristique de l'amortissement $D$ du sol (Richart et al., 1970; Auersch, 1994), qui est défini par :

$$
G=G_{0}(1+i 2 D)
$$

et qui est considéré croissant avec la fréquence (allant de $D=1 \%$ à $10 \mathrm{~Hz}$ jusqu'à $D$ $=10 \%$ à $100 \mathrm{~Hz}$ ) pour ce site.

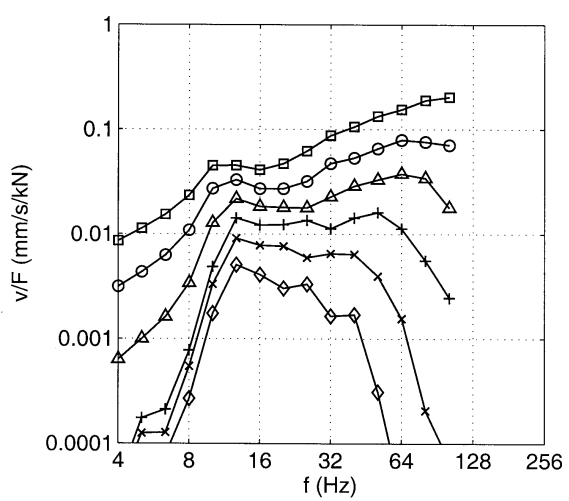

(a)

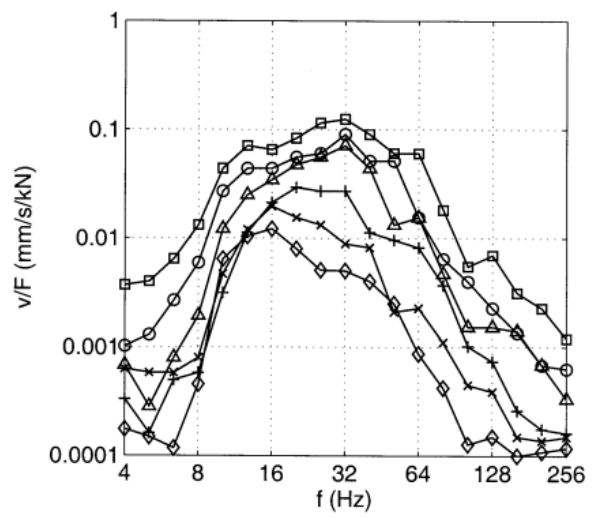

(b)

Figure 2. Fonction de transfert du sol à Würzburg : (a) calculs, sol constitué d'une première couche $\left(v_{S}=250 \mathrm{~m} / \mathrm{s}, h=10 \mathrm{~m}\right)$, et reposant sur un sous-sol raide ( $v_{S}=$ $1000 \mathrm{~m} / \mathrm{s})$, amortissement croissant avec la fréquence $(D=1$ à $10 \%)$, distance du point d'excitation $r=\square 2, O 4, \triangle 8,+16, \times 32, \diamond 64 m-(b)$ comparaison avec les mesures, distance du point d'excitation $r=\square 2.5,05, \triangle 10,+20, \times 30 \mathrm{~m}, \diamond 45 \mathrm{~m}$

Les amplitudes pour les fréquences plus basses que $f=12 \mathrm{~Hz}$ sont réduites de façon significative. Cette « fréquence de coupure » en basses fréquences est une ca- 
ractéristique d'un sol ayant une première couche souple et reposant sur un massif beaucoup plus rigide et peut être estimée par :

$$
f \approx \frac{v_{S}}{2 d} \approx \frac{v_{P}}{4 d}
$$

voir Wolf (1985). Pour les fréquences moyennes les amplitudes augmentent légèrement, et aux fréquences hautes les mobilités sont proches de celles du sol homogène $\left(v_{S}=250 \mathrm{~m} / \mathrm{s}\right)$. Le rôle de la première couche souple est d'autant plus important que le contraste des vitesses de l'onde de la couche et du massif est fort (Auersch, 1994). Les fonctions de transfert mesurées près de Würzburg (figure 2|b)) sont relativement bien représentées par ce modèle de sol.

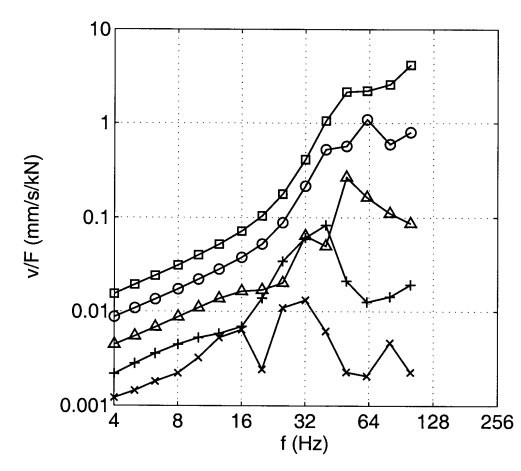

(a)

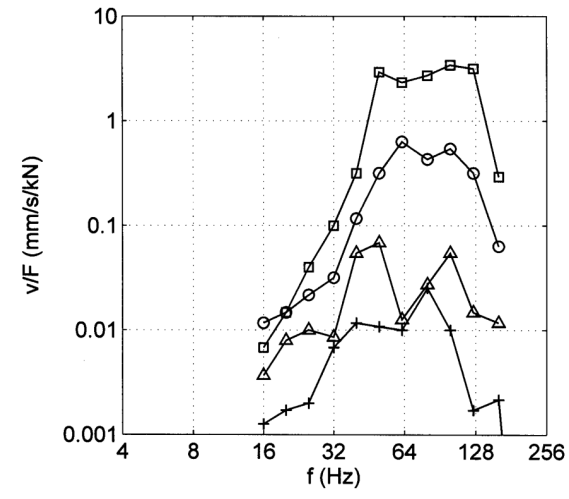

(b)

Figure 3. Fonction de transfert du sol à Nantes : (a) calculs en considérant deux couches $\left(v_{S}=77\right.$ et $\left.111 \mathrm{~m} / \mathrm{s}\right)$ d'épaisseurs 0,4 et $0,6 \mathrm{~m}$, d'amortissements $7,5 \%$ et 5 $\%$, reposant sur un massif plus raide $\left(v_{S}=208 \mathrm{~m} / \mathrm{s}\right)$ avec un amortissement $3 \%-(b)$ mesures - distances du point d'excitation $r=\square 2,04, \triangle 8,+16, \times 32 \mathrm{~m}$

Le modèle pour le sol de Nantes inclut deux couches sur un massif semi-infini (sous-sol), avec des vitesses de cisaillement valant respectivement 77, 111 et $208 \mathrm{~m} / \mathrm{s}$. Les épaisseurs des deux couches sont de 0,4 et 0,6 m, et les amortissements recalés valent 7,5 et $5 \%$ pour les deux couches et $3 \%$ sur le massif semi-infini. Les autres paramètres du site de Nantes sont les suivants :

$$
\begin{array}{r}
\rho_{1}=1600 \mathrm{~kg} / \mathrm{m}^{3} \quad \rho_{2}=1700 \mathrm{~kg} / \mathrm{m}^{3} \quad \rho_{3}=1800 \mathrm{~kg} / \mathrm{m}^{3} \\
\nu_{1}=0.4 \quad \nu_{2}=0.35 \quad \nu_{3}=0.33
\end{array}
$$

Les fonctions de transfert théoriques (figure 3(a)) correspondent assez bien aux fonctions expérimentales qui sont mesurées suivant une direction parallèle à la voie pour des distances allant de 2 à $16 \mathrm{~m}$ (figure 3 (b)). Les amplitudes de ce sol très 
souple sont environ dix fois plus grandes que celles du site de Würzburg notamment au-delà de $40 \mathrm{~Hz}$. Pour les fréquences plus basses que cette « fréquence de coupure », on remarque que les amplitudes sont réduites. Enfin, la décroissance des amplitudes vibratoires pour les fréquences élevées (au-delà de $100 \mathrm{~Hz}$ ) est due à l'amortissement fort sur la première couche $(\mathrm{D}=7.5 \%)$.

\section{Analyse de la fonction de transfert voie-sol et des réductions par la voie}

La fonction de transfert voie-sol théorique (équation [18]) est calculée pour la voie avec une dalle de béton. Celle-ci a les caractéristiques suivantes :

$$
\begin{array}{ll}
2 a=3 \mathrm{~m} & \text { largeur, } \\
d_{D}=0.6 \mathrm{~m} & \text { épaisseur, } \\
E=3 \cdot 10^{10} \mathrm{~N} / \mathrm{m}^{2} & \text { module de Young, } \\
\rho=2500 \mathrm{~kg} / \mathrm{m}^{3} & \text { masse volumique. }
\end{array}
$$

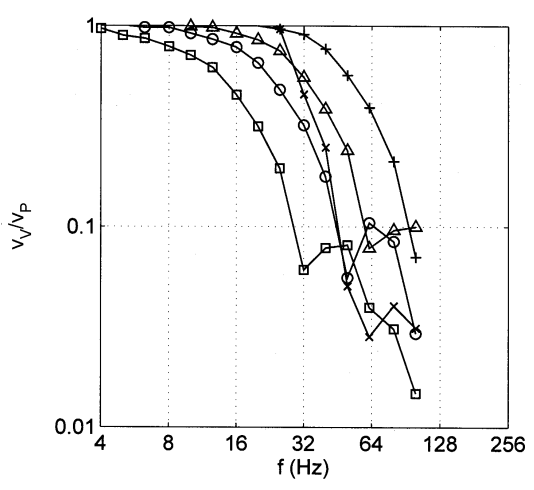

(a)

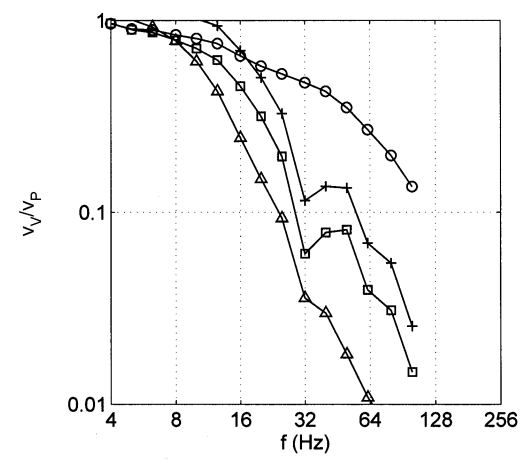

(b)

Figure 4. Réduction des vibrations par la voie : amplitudes de vibrations du sol d'une source sur la voie $v_{V}$ divisées par les amplitudes dues à la source ponctuelle $v_{P}$. (a) Calculs pour les sols $v_{S}=\square 100, O 150, \triangle 200,+300 \mathrm{~m} / \mathrm{s}, \times$ sol à Nantes $-(b)$ sol souple $\left(v_{S}=100 \mathrm{~m} / \mathrm{s}\right)$, calculs pour une $\square$ voie sur dalle, $O$ voie étroite $(2 a=0,5 \mathrm{~m})$, $\triangle$ masse double, + aucune rigidité

La figure 4 a) présente la réduction des vibrations par la voie sur dalle pour différents sols (sol de Nantes et sols avec $v_{S}=100,150,200$ et $300 \mathrm{~m} / \mathrm{s}$ ). Les amplitudes de vibrations du sol dues à une source d'excitation sur la voie $v_{V}$ sont divisées par les amplitudes dues à une source ponctuelle $v_{P}$. Les réductions ont des allures similaires mais elles commencent à des fréquences différentes. Lorsque le sol est plus souple ( $v_{S}$ 


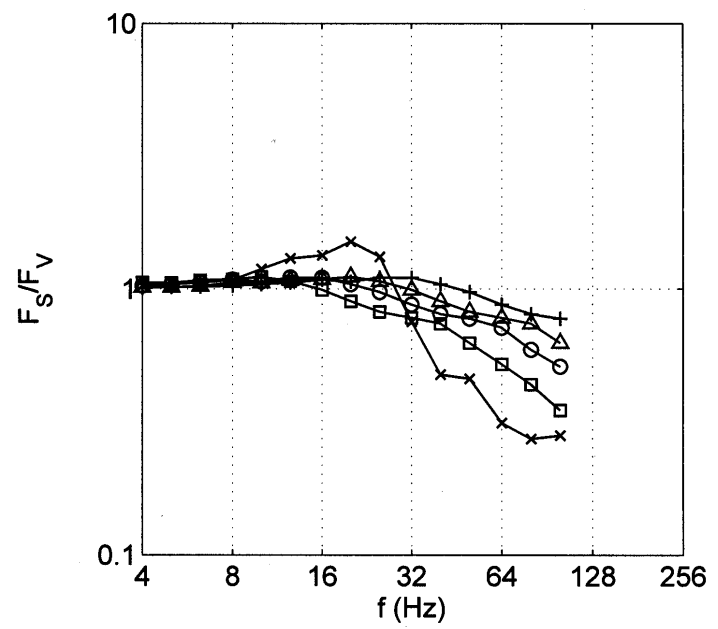

Figure 5. Réduction des efforts par la voie sur dalle béton, rapport de la force à l'interface dalle/sol $F_{S}$ sur la force à l'interface roue/rail $F_{V}$, calculs pour les sols homogènes $v_{S}=\square 100, O 150, \triangle 200,+300 \mathrm{~m} / \mathrm{s}$ et $\times$ le sol de Nantes

$=100 \mathrm{~m} / \mathrm{s}$ ), la réduction commence à des fréquences plus basses et la zone fréquentielle de la réduction est plus large. Au bilan, la réduction est d'autant plus efficace que le sol est souple.

Les effets de la rigidité, de la masse et de la largeur de la voie sont présentés sur la figure 4(b) en comparaison avec la voie sur dalle de Nantes. Si on prend une masse double, la réduction est très forte. Si la voie n'a aucune rigidité, la réduction de la voie est un peu moins bonne. Si la force est distribuée sur une voie étroite $(2 a=0,5$ $\mathrm{m})$ la réduction est faible. Inversement, la réduction obtenue avec une voie « large » $(2 a=3 \mathrm{~m})$ est forte. Ces différents effets sont dus à l'atténuation de la force dynamique par la masse de la voie et à la distribution de la force agissant entre la voie et le sol. La fonction de transfert voie/sol des efforts correspondant au rapport de la force à l'interface dalle/sol $F_{S}$ sur la force à l'interface roue/rail $F_{V}$ est présentée sur la figure 5 pour les différents sols. On constate des réductions inférieures pour les sols homogènes. Pour la situation de Nantes avec des couches différentes, l'amortissement réduit induit des fortes variations de la fonction de transfert : résonance autour de $20 \mathrm{~Hz}$ et forte réduction aux hautes fréquences. Tous ces calculs montrent que la réduction due à la distribution des forces au travers de la voie joue un rôle crucial pour la propagation des vibrations. Précisément, les paramètres les plus importants sont la largeur de la voie et la vitesse de l'onde à la surface du sol. 


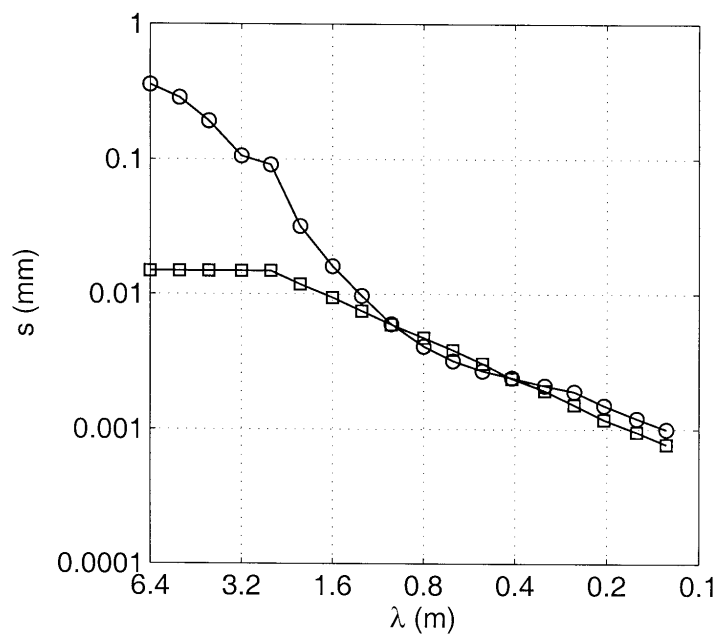

Figure 6. Irrégularités de la voie et des roues combinées et simplifiées, $\square$ Nantes et O Würzburg

\section{Interaction véhicule-voie, calcul des forces d'interaction}

\subsection{Irrégularités de la voie et des roues}

Les efforts liés à l'interaction véhicule-voie-sol sont générés par les irrégularités des rails et des roues. Les irrégularités des rails ont été mesurées pour le site de Nantes, voir Maldonado (2008). Les irrégularités des roues n'ont pas été mesurées directement, mais les accélérations mesurées sur un essieu montrent que ces irrégularités sont non négligeables seulement pour les fréquences supérieures à $100 \mathrm{~Hz}$. Des rugosités combinées (roues et rails) et simplifiées sont construites avec les irrégularités mesurées et les accélérations verticales des essieux (figure 6) et utilisées pour les comparaisons suivantes. Les irrégularités combinées de Würzburg sont évaluées de façon similaire par les accélérations des roues (Auersch, 2005b; Auersch, 2010), voir figure 6 Les irrégularités de Nantes et Würzburg sont presque les mêmes pour les longueurs d'onde courtes. Les irrégularités pour longueurs d'ondes longues pour la voie sur dalle sont plus faibles que celles obtenues pour la voie ballastée. Ceci montre sans doute la plus grande efficacité de la pose sur dalle.

Pour les calculs de l'interaction véhicule-voie il faut exprimer les irrégularités en fonction de la fréquence $f$ :

$$
s(f)=s\left(\lambda=\frac{v_{T}}{f}\right)
$$


Ces résultats sont présentés sur la figure 7 pour les vitesses $v_{T}=40 \mathrm{~km} / \mathrm{h}$ à Nantes et $v_{T}=100 \mathrm{~km} / \mathrm{h}$ à Würzburg. Les irrégularités diminuent avec la fréquence.

\subsection{Fonction de transfert véhicule-voie}

Les raideurs dynamiques de la voie et du véhicule (équations [21] et [27]) sont combinées pour obtenir la fonction de transfert [26] de l'interaction véhicule-voie. La masse des roues $m_{R}=1500 \mathrm{~kg}$ est la même pour les deux situations en France et en Allemagne. Les raideurs dynamiques de la voie diffèrent fortement (tableau 2).

\begin{tabular}{|l|l|l|}
\hline Paramètres & Voie de Nantes & Voie de Würzburg \\
\hline Rail, raideur $E I$ & $4.3 \cdot 10^{6} \mathrm{Nm}^{2}$ & $6.4 \cdot 10^{6} \mathrm{Nm}^{2}$ \\
Rail, masse $m^{\prime}$ & $55 \mathrm{~kg} / \mathrm{m}$ & $60 \mathrm{~kg} / \mathrm{m}$ \\
Semelles, raideur & $90 \cdot 10^{6} \mathrm{~N} / \mathrm{m}^{2}$ & $300 \cdot 10^{6} \mathrm{~N} / \mathrm{m}^{2}$ \\
Travelage & $0.75 \mathrm{~m}$ & $0.6 \mathrm{~m}$ \\
Largeur & $3.0 \mathrm{~m}$ (dalle) & $2.6 \mathrm{~m}$ (traverse) \\
Épaisseur & $0.6 \mathrm{~m}$ (dalle) & $0.35 \mathrm{~m}$ (ballast) \\
Matériau & béton (voir section 5) & $\begin{array}{l}\text { ballast modélisé par la première } \\
\text { couche du sol (voir section 4) }\end{array}$ \\
\hline
\end{tabular}

Tableau 2. Paramètres des voies à Nantes et Würzburg

La voie ballastée à Würzburg est très rigide parce que le ballast est très rigide pour cette ligne à grande vitesse tout comme le sol et les semelles des rails. La voie sur dalle à Nantes est globalement plus souple parce que les semelles des rails sont assez souples. Les fonctions de transfert des deux voies sont présentées sur la figure 7. Elles sont croissantes jusqu'à la résonance de l'essieu sur la voie, autour de $64 \mathrm{~Hz}$ pour la voie de Nantes et de $100 \mathrm{~Hz}$ pour la voie de Würzburg.

\subsection{Forces d'interaction entre le véhicule et la voie}

La fonction de transfert théorique entre la voie et le véhicule, obtenue par le biais des irrégularités, donne les forces d'interaction (forces dynamiques au contact rouerail). Ces forces sont présentées sur la figure 8 . Elles sont croissantes pour les fréquences basses et maximales à $64 \mathrm{~Hz}$ pour Nantes.

Les forces d'interaction peuvent aussi être calculées à l'aide des accélérations mesurées sur un essieu. Il faut alors multiplier les accélérations par la raideur dynamique du véhicule (équation [27]). Ces forces estimées à l'aide des accélérations mesurées sont aussi données sur la figure 8 Elles sont comparables aux forces déduites des rugosités. Les amplitudes plus élevées entre 128 et $160 \mathrm{~Hz}$ sont probablement dues à un mode élastique d'essieu. Il apparait que les forces calculées avec les irrégularités sont plus régulières. 


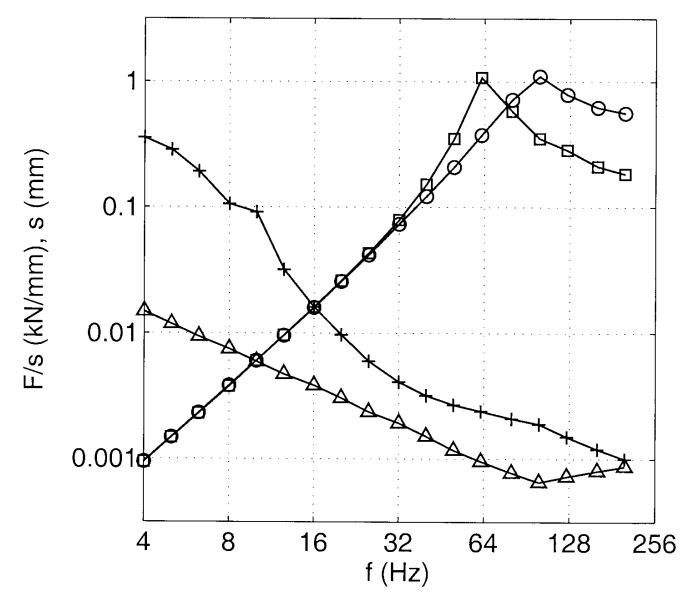

Figure 7. Fonction de transfert d'interaction véhicule-voie (rapport de la force $F$ au contact roue-rail sur la rugosité s), $\square$ Nantes et $O$ Würzburg, et irrégularités de la voie et des roues combinées et simplifiées, $\triangle$ Nantes et + Würzburg

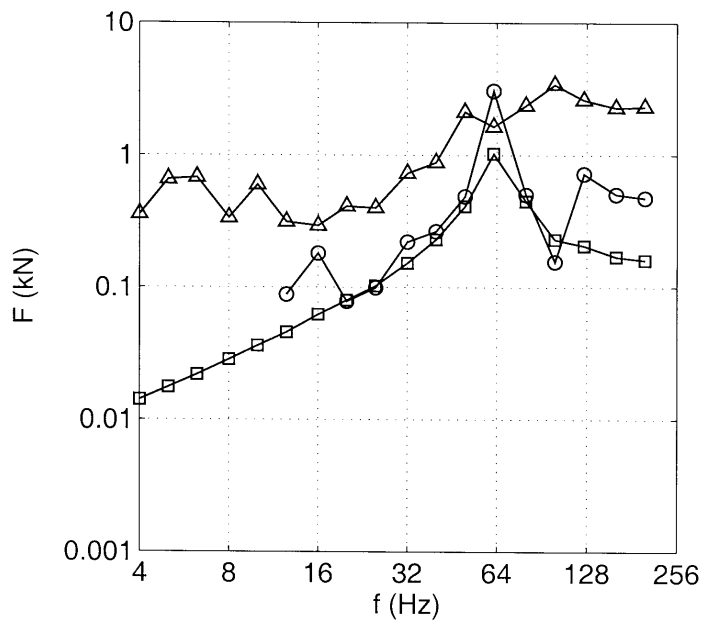

Figure 8. Forces roue-voie calculées à l'aide des accélérations d'essieux (mesures), $O$ Nantes et $\triangle$ Würzburg, et calculées à l'aide des rugosités combinées et simplifiées (modèle) $\square$ Nantes 


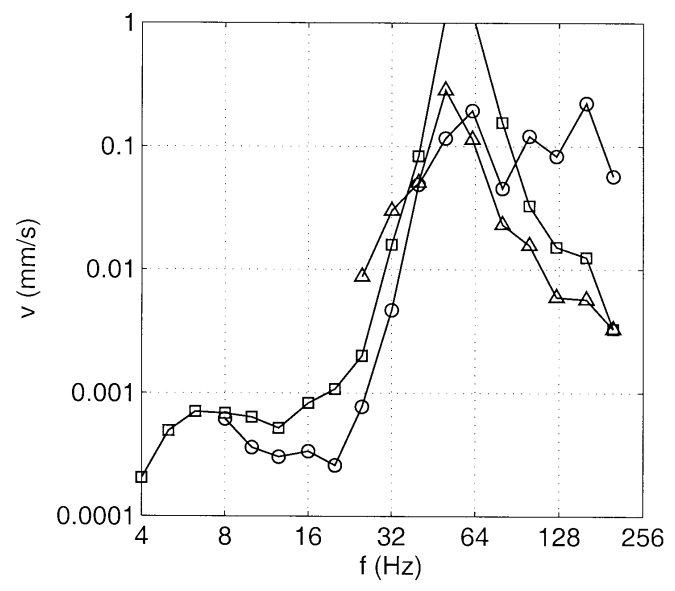

Figure 9. Vibrations du sol à une distance de $2 \mathrm{~m}$ de la voie, passage d'un tramway à $40 \mathrm{~km} / \mathrm{h}$, O mesurées, $\triangle$ complètement calculées (Maldonado, 2008), $\square$ calculées avec la fonction de transfert voie-sol mesurée

Pour la situation à Würzburg, les forces sont aussi calculées en se basant sur des mesures d'accélérations d'essieu. Elles sont plus grandes que les forces obtenues pour le tramway (Nantes), presque constantes autour de $1 \mathrm{kN}$ (Auersch, 2010), et les amplitudes aux hautes fréquences sont légèrement plus élevées.

\section{Vibrations du sol dues au passage du train}

\subsection{Le tramway à Nantes}

Les vibrations du sol pendant le passage d'un train peuvent être calculées à l'aide des forces d'interaction véhicule-voie (figure 8, section 6.3). Pour les passages d'un tram au centre-ville de Nantes, il était seulement possible d'installer les capteurs relativement proches de la voie. La figure 9 montre les résultats a une distance de $2 \mathrm{~m}$ du rail extérieur. Sur la même figure le calcul théorique est présenté (équation [28], Maldonado (2008)). La conformité entre les résultats théoriques et expérimentaux est très bonne pour les fréquences moyennes. Aux fréquences hautes les amplitudes mesurées sont plus fortes. Pour comparaison, la prédiction avec la fonction de transfert mesurée est représentée par la courbe $\square$. Ce résultat montre aussi des amplitudes très réalistes aux fréquences basses. Les amplitudes maximales du sol à 50 et $64 \mathrm{~Hz}$ sont mieux estimées à l'aide du modèle complet, et les amplitudes aux hautes fréquences sont sous-évaluées pour les deux prédictions. 


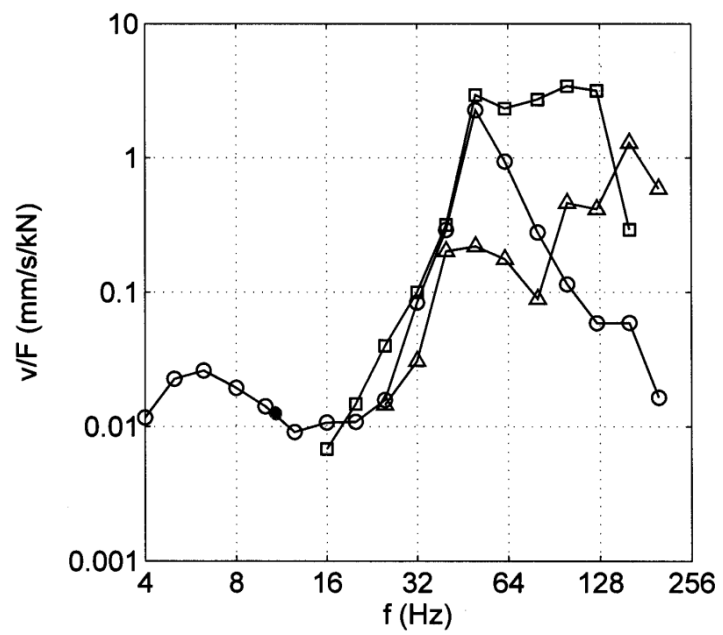

Figure 10. Fonctions de transfert $\square d u$ sol, $O$ voie-sol et $\triangle$ train-sol à la distance de 2 m mesurées à Nantes

Les vibrations du sol mesurées pendant le passage d'un train peuvent être utilisées pour établir une autre fonction de transfert, la fonction de transfert train-sol (définie par l'équation [28]). Les amplitudes du sol mesurées sont alors divisées par les forces d'interaction. Les résultats sont présentés sur la figure 10 avec les mobilités ponctuelle du sol (définies par l'équation [11]) et de transfert voie-sol (définie par l'équation [18]). On constate pour les trois fonctions la réduction des amplitudes pour les fréquences basses qui est une caractéristique du site de Nantes.

\subsection{Le train à Würzburg}

Les vibrations du sol dues à un train se déplaçant à $100 \mathrm{~km} / \mathrm{h}$ sont calculées avec les forces mesurées présentées sur la figure 8 et avec la fonction de transfert théorique de la figure 2(a). La sommation des contributions des forces provenant de tous les essieux du train fournit les amplitudes du sol calculées (figure 11). L'atténuation avec la distance est très régulière et plus faible pour le train que l'atténuation d'une source ponctuelle.

La prédiction des vibrations du sol est aussi possible avec la fonction de transfert mesurée présentée sur la figure 2 b). Les amplitudes du sol (figure 12) sont très similaires comme celles de la prédiction théorique (figure 11) mais l'atténuation est plus faible notamment aux fréquences hautes. La comparaison avec les amplitudes mesurées sur le sol pendant le passage d'un train (figure-13) montre un très bon accord avec les amplitudes prédites. Finalement, le comportement en champ proche de la voie et 


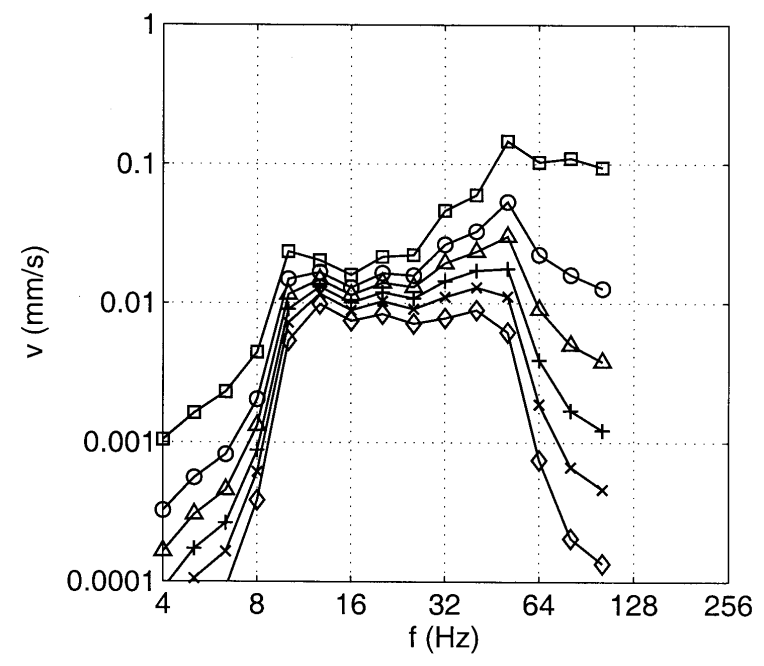

Figure 11. Passage d'un train à grandes lignes à Würzburg, vibrations du sol calculées pour les distances $r=\square 2.5, O 7.5, \triangle 12.5,+20, \times 30 \mathrm{~m}, \diamond 50 \mathrm{~m}$

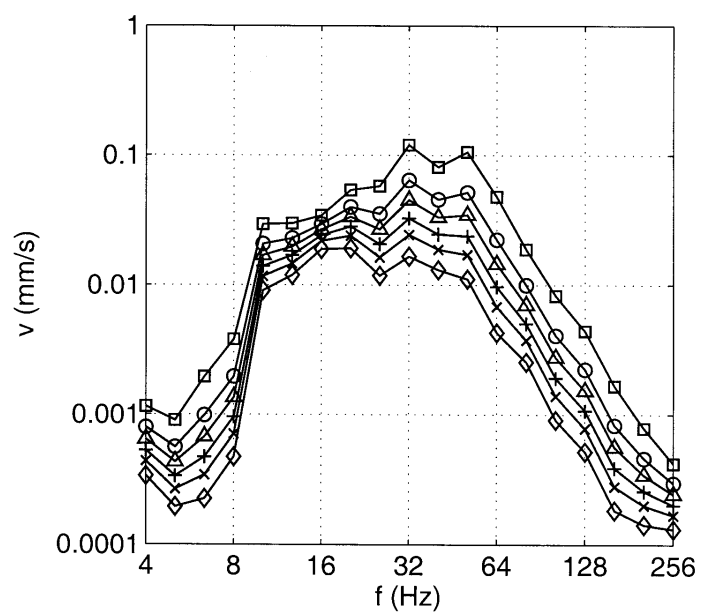

Figure 12. Passage d'un train à grandes lignes à Würzburg, vibrations du sol calculées (avec la fonction de transfert du sol mesurée) pour les distances $r=\square 2.5, O$ 7.5, $\triangle 12.5,+20, \times 30 \mathrm{~m}, \diamond 50 \mathrm{~m}$ 


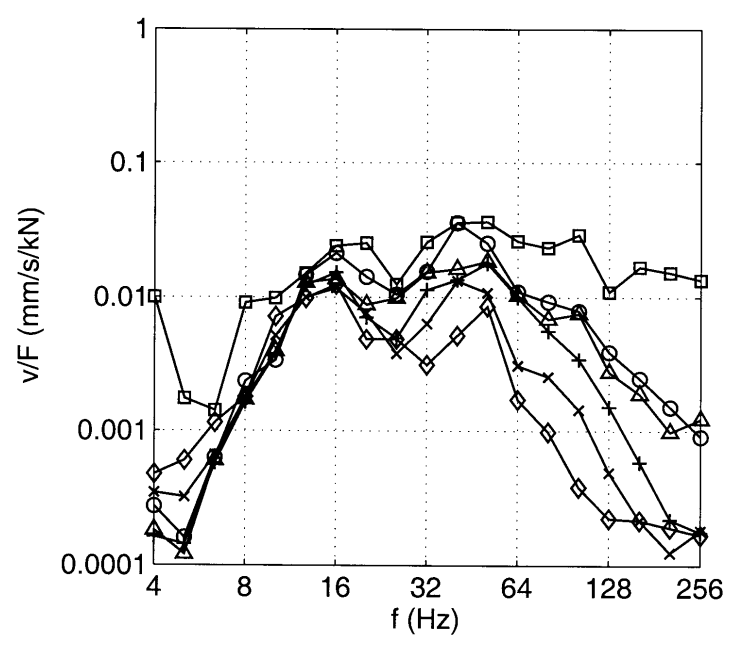

Figure 13. Passage d'un train à grandes lignes à Würzburg, vibrations du sol mesurées pour les distances $r=\square 2.5, O 7.5, \triangle 12.5,+20, \times 30 \mathrm{~m}, \diamond 50 \mathrm{~m}$

l'atténuation forte aux fréquences basses et hautes sont mieux estimés en utilisant la fonction de transfert théorique plutôt que mesurée.

\section{Conclusion}

Les différents aspects liés aux vibrations dues aux trains sont abordés selon une approche globale de l'interaction véhicule-voie-sol. Les vibrations du sol et de la voie sont calculées par une intégration numérique dans le domaine des nombres d'onde. L'influence importante de l'amortissement et des caractéristiques des couches du sol est mise en évidence à l'aide des modèles du sol pour deux sites expérimentaux en France et en Allemagne. Concernant les amplitudes du sol, une réduction importante provient de la voie, elle dépend principalement de la largeur de la voie et de la vitesse de l'onde de surface. Différents paramètres pour l'excitation des vibrations sur le sol sont étudiés : la réceptance de la voie, la raideur dynamique du véhicule, la fonction de transfert véhicule-voie, les irrégularités de la voie et des roues, et les forces rouerail, qui résultent des irrégularités. Les paramètres physiques en entrée des modèles sont vérifiés par de nombreuses mesures, sur les sols, les voies, et lors du passage des véhicules (tramways à Nantes et trains à passagers à Würzburg). Il faut souligner que les forces d'interaction véhicule-voie estimées par calculs et prises en compte pour obtenir les vibrations du sol donnent des résultats satisfaisants vis-à-vis des vibrations mesurées. Ceci confirme que la génération des vibrations provient principalement de l'interaction roue-rail, liée aux irrégularités dans le contact. Les forces générées par 
le tramway sont inférieures à celles dues au train à passagers. Mais, étant donné que le sol est plus souple là où circule le tramway, les vibrations du sol sont comparables pour les deux trains sur des sols pourtant différents. Au final, ces résultats numériques et expérimentaux montrent bien l'influence importante du sol vis-à-vis des vibrations dues aux trains.

\section{Remerciements}

L. Auersch remercie S. Said et W. Schmid pour leur coopération agréable pendant les mesures à Würzburg. M. Maldonado remercie O. Chiello pour les mesures faites à Nantes, fruit d'une collaboration entre l'INRETS et l'Ecole Centrale.

\section{Bibliographie}

Adolfsson K., Andreasson B., Bengtsson P., Zackrisson P., « Highspeed train X2000 on soft organic clay - measurements in Sweden », Proc. 12th Europ. Conf. Soil Mechanics and Geotechnical Engineering, Balkema, Rotterdam, p. 1713-1718, 1999.

Aubry D., Clouteau D., Bonnet G., « Modeling of wave propagation due to fixed or mobile dynamic sources », Proc. WAVE'94 Wave Propagation and Reduction of Vibrations, Bochum, p. 109-121, 1994.

Auersch L., Zur Entstehung und Ausbreitung von Schienenverkehrserschütterungen : Theoretische Untersuchungen und Messungen am Hochgeschwindigkeitszug Intercity Experimental, Research report 155, BAM Berlin, 1988.

Auersch L., « Parametric excitation of rail-wheel-system : calculation of vehicle-track-subsoildynamics and experimental results of the high speed train Intercity Experimental », Archive of Applied Mechanics, vol. 60, p. 141-156, 1990. (en allemand).

Auersch L., « Wave propagation in layered soil : theoretical solution in wavenumber domain and experimental results of hammer and railway traffic excitation », Journal of Sound and Vibration, vol. 173, p. 233-264, 1994. doi 10.1006/jsvi.1994.1228

Auersch L., " Dynamics of the railway track and the underlying soil : the boundary-element solution, theoretical results and their experimental verification », Vehicle System Dynamics, vol. 43, p. 671-695, 2005a.

Auersch L., " The excitation of ground vibration by rail traffic : Theory of vehicle-track-soil interaction and measurements on high-speed lines », Journal of Sound and Vibration, vol. 284, p. 103-132, 2005b. doi $10.1016 /$ j.jsv.2004.06.017.

Auersch L., « Dynamic interaction of various beams with the underlying soil - finite and infinite, half-space and Winkler models », European Journal of Mechanics - A/Solids, vol. 27, p. 933-958, 2008a. doi 10.1016/j.euromechsol.2008.02.001

Auersch L., « The effect of critically moving loads on the vibrations of soft soils and isolated railway tracks », Journal of Sound and Vibration, vol. 310, p. 587-607, 2008b. doi $10.1016 /$ j.jsv.2007.10.013 
Auersch L., « Theoretical and experimental excitation force spectra for railway induced ground vibration - vehicle-track soil interaction, irregularities and soil measurements », Vehicle System Dynamics, vol. 48, p. 235-261, 2010.

Auersch L., Said S., Rücker W., Das Fahrzeug-Fahrweg-Verhalten und die Umgebungserschütterungen bei Eisenbahnen, Forschungsbericht 243, BAM Berlin, 2001.

Barros F., Luco J., « Response of a layered visco-elastic half-space to a moving point load », Wave Motion, vol. 19, p. 189-210, 1994.

Dietermann H., Metrikine A., « The equivalent stiffness of a half-space interacting with a beam. Critical velocities of a load moving along a beam », European Journal of Mechanics A/Solids, vol. 15, p. 67-90, 1996.

Fröhling R., Deterioration of railway track due to dynamic vehicle loading and spatially varying track stiffness, Thèse de doctorat, University of Pretoria, 1997.

Fryba L., Vibrations of Structures under Moving Loads, Noordhof Int. Publishing, Groningen, 1972.

Fujikake T., «A prediction method for the propagation of ground vibration from railway trains », Journal of Sound and Vibrations, vol. 111, p. 357-360, 1986.

Galvin P., Dominguez J., « Experimental and numerical analyses of vibrations induced by highspeed trains on the Córdoba-Málaga line », Soil Dynamics and Earthquake Engineering, vol. 29, p. 641-657, 2009. doi 10.1016/j.soildyn.2008.07.001

Girardi L., « Propagation des vibrations dans les sols homogènes ou stratifiés », Annales de lÍnstitut Technique du Batiment et Travaux Public, vol. 397, p. 30-65, 1981.

Grundmann H., Lieb M., Trommer E., « The response of a layered half-space to traffic loads moving along its surface », Archive of Applied Mechanics, vol. 69, p. 55-67, 1999. doi $10.1007 / \mathrm{s} 004190050204$

Hanson C., Towers D., Meister L., Transit noise and vibration impact assessment, Report ftava-90-1003-06 for the federal transit administration, HMMH Inc., Burlington, 2006.

Huber G., Erschütterungsausbreitung beim Rad/Schiene-System, Thèse de doctorat, University Karlsruhe, 1988.

Hung H., Yang Y., « A review of researches on ground-borne vibrations with emphasis on those induced by trains », Proc. Natl. Sci. Counc. ROC(A), vol. 25, p. 1-16, 2001.

Jones C., Block J., « Prediction of ground vibration from freight trains », Journal of Sound and Vibration, vol. 193, p. 205-213, 1996.

Jones D., Surface propagation of ground vibration, Thèse de doctorat, University Southampton, 1987.

Kausel E., Roesset J., « Stiffness matrices for layered soils », Bulletin of the Seismological Society of America, vol. 71, p. 1743-1761, 1981.

Knothe K., Grassie S., « Modelling of railway track and vehicle/track interaction at high frequencies », Vehicle System Dynamics, vol. 22, p. 209-262, 1993.

Krylov V., Generation of ground vibration boom by high-speed trains, V. Krylov (ed.) Noise and Vibration from High-Speed Trains, Telford, London, 2001.

Laghrouche O., Simulation numérique de propagation d'ondes dans les sols, application à l'isolation vibratoire, Thèse de doctorat, Université de Nantes, École Centrale de Nantes, 1996.

Lai C., Rix G., Foti S., Roma V., « Simultaneous Measurement and inversion of surface wave dispersion and attenuation curves », Soil Dyn. Earthq. Eng., vol. 22, p. 923-930, 2002. 
Lefeuve-Mesgouez G., Propagation d'ondes dans un massif soumis à des charges se déplaçant à une vitesse constante, Thèse de doctorat, Université de Nantes, École Centrale de Nantes, 1999.

Lombaert G., Degrande G., Kogut J., François S., « The experimental validation of a numerical model for the prediction of railway induced vibrations », Journal of Sound and Vibration, vol. 297, p. 512-535, 2006. doi $10.1016 /$ j.jsv.2006.03.048

Madshus C., Kaynia A., « High speed railway lines on soft ground, dynamic behaviour at critical speed », Journal of Sound and Vibration, vol. 231, p. 689-701, 2000. doi $10.1006 /$ jsvi.1999.2647

Maldonado M., Vibrations dues au passage d'un tramway - mesures expérimentales et simulations numériques, Thèse de doctorat, École Centrale de Nantes, 2008. http://tel. archives-ouvertes.fr/tel-00356222/fr/

Maldonado M., Pallas M., Philipps-Bertin C., Bruit et vibrations dus aux tramways : émission et perception, Rapport INRETS n 279 , Ed. Lavoisier, 2009.

Nelson J., Saurenman H., « A prediction procedure for rail transportation groundbourne noise and vibration », Transportation Research Record, vol. 1143, p. 26-35, 1987.

Nielsen J., Train-track interaction. Coupling of moving and stationary systems - theoretical and experimental analysis of railway structures considering wheel and track imperfections, Thèse de doctorat, Chalmers University of Technology Göteborg, 1993.

O’Brien J., Rizos D., « A 3D BEM-FEM methodology for simulation of high speed train induced vibrations », Soil Dynamics and Earthquake Engineering, vol. 25, p. 289-301, 2005.

Picoux B., Étude théorique et expérimentale de la propagation dans le sol des vibrations émises par un traffic ferroviaire, Thèse de doctorat, Université de Nantes, École Centrale de Nantes, 2002.

Richart F., Hall J., Woods R., Vibration of Soils and Foundations, Prentice-Hall, New Jersey, 1970.

Saedeleer B., Bilon S., Datoussaid S., Conti C., « Vehicle/track interaction and ground propagation of vibrations for tramway tracks in urban areas », Proc. Int. Workshop on Railway Vibrations and Rail Vehicle Dynamics, Barcelona, p. 39-62, 1998.

Sheng X., Ground vibration generated from trains, Thèse de doctorat, University of Southampton, 2001.

Takemiya H., « Simulation of track-ground vibrations due to high-speed train : the case of X-2000 at Ledsgard», Journal of Sound and Vibration, vol. 261, p. 503-526, 2003. doi $10.1016 / \mathrm{S} 0022-460 \mathrm{X}(02) 01007-6$.

van den Broek P., A prediction model for ground-borne vibrations due to railway traffic, Thèse de doctorat, KU Leuven, 2001.

Wolf J., Dynamic Soil-Structure Interaction, Prentice-Hall, New Jersey, 1985.

Yokoyama H.and Ashiya K., Iwata N., « An evaluation method for train-speed dependency of Shinkansen-induced vibration », In Takemiya, H. (Ed.) Environmental Vibrations, Okayama, p. 345-350, 2005.

Reçu le : 1er mars 2010 Accepté le : 1er mars 2011 